\title{
Thermal Vibration of Magnetostrictive Material in Laminated Plates by the GDQ Method
}

\author{
C.C. Hong
}

Department of Mechanical Engineering, Hsiuping Institute of Technology, Taichung, 412 Taiwan, ROC

\begin{abstract}
The study of magnetostrictive material in laminated plate under thermal vibration is calculated by using the generalized differential quadrature (GDQ) method. The YNS shear deformation effect is included in the time dependent of displacement field. In the thermoelastic stress-strain relations that containing the linear temperature rise and the magnetostrictive coupling terms with velocity feedback control. We use the GDQ method to normalize and discrete the dynamic differential equations in terms of displacements and shear rotations into the form of dynamic discretized equations. Four edges of rectangular laminate with simply supported boundary conditions are considered. The time responses of thermal stresses and center displacement with and without velocity control are obtained.
\end{abstract}

Keywords: Magnetostrictive material, thermal vibration, generalized differential quadrature method, GDQ, shear deformation, velocity feedback control.

\section{INTRODUCTION}

One of the new trends of material in the mechanical engineering is the functionally gradient materials (FGM). In each type of FGM, there are some functional layers e.g. piezoelectric, magnetostrictive, electrostrictive, shape memory alloys, in their sections can make a controlled progressive change of smart structures [1,2]. Magnetostrictive materials have the Magneto-electric coupling property under the action of magnetism and mechanism. Terfenol-D is one of available Magnetostrictive materials, can be used usually in the sensors and actuators [3].

In 2005, Lee and Reddy presented the finite element method to analyze the non-linear response of laminated plate of Magnetostrictive material under thermo-mechanical loading [3]. They found the temperature can decrease the amplitude and period of deflection. In 2005, Pradhan presented the analytical solutions for the FGM shells of embedded Magnetostrictive layers under vibration [1].

It was found that the Magnetostrictive layers should be placed away from the neutral plane and made thinner to get better attenuation effects. In 2006, Ramirez, Heyliger and Pan present the Ritz approach to get an approximate solution for the free vibration problem of two-dimensional magnetoelectro-elastic laminates [4]. They obtained the elastic displacements, electric potential, and magnetic potential numerical solutions. In 2004, Buchanan presented the candidate materials of multiphase magneto-electro-elastic composites [5]. They found the multiphase material properties vary depending upon the ratio of fiber material to matrix material. In 2003, Hong and Jane presented the GDQ method to study the shear deformation in the thermal vibration and bending of laminated plates [6,7]. They found that the maximum deflection and interlaminar stresses at center posi-

*Address correspondence to this author at the Department of Mechanical Engineering, Hsiuping Institute of Technology, Taichung, 412 Taiwan, ROC; E-mail: cchong@mail.hit.edu.tw tion of laminate increasing with the side-to-thickness ratio value decreasing. In 2005, Hong et al. presented the GDQ method to study the thermal vibration of a thermal sleeve [8]. They found that the computational GDQ solutions of the natural frequency, displacement and thermal stress. In 2006, Hong et al. used the GDQ method to study the piezoelectric material under mechanical and electric loads [9]. They found that the numerical GDQ solutions of stress and electric potential function for the analyses of local symmetric pressure with no overshoot and no Gibbs effect and under tractionfree boundary condition of the strip. Now it is interesting to study and find the natural frequency, displacement and thermal stress of the Magnetostrictive material in laminated plates with the GDQ method.

\section{FORMULATION}

\subsection{Displacement Field}

The time dependent of displacements fields are assumed in the following YNS (Yang-Norris-Stavsky, 1966) form [6], which is the first-order shear deformation theory in the linear equation:

$$
\begin{aligned}
& u=u^{0}(x, y, t)+z \psi_{x}(x, y, t) \\
& v=v^{0}(x, y, t)+z \psi_{y}(x, y, t) \\
& w=w(x, y, t)
\end{aligned}
$$

where $u^{0}$ and $v^{0}$ are tangential displacements, $w$ is transverse displacement of the middle-plane, $\psi_{x}$ and $\psi_{y}$ are the shear rotations, $t$ is time.

\subsection{GDQ Method}

The GDQ method was presented in 1990 by Shu and Richards [11]. The GDQ method approximates the derivative of function would be used [6] and can be restated that: the derivative of a smooth function at a discrete point in a domain can be discretized by using an approximated weighting linear sum of the function values at all the discrete points in the direction $[10,11]$. For example, the first-order and the 
second-order derivatives of function $f^{*}(x, y)$ at coordinates $\left(x_{i}, y_{j}\right)$ of grid point $(i, j)$ can be discretized by:

$\left.\frac{\partial f^{*}}{\partial x}\right|_{i, j} \approx \sum_{l=1}^{N} A_{i, l}^{(1)} f_{l, j}^{*},\left.\frac{\partial f^{*}}{\partial y}\right|_{i, j} \approx \sum_{m=1}^{M} B_{j, m}^{(1)} f_{i, m}^{*}$,

$\left.\frac{\partial^{2} f^{*}}{\partial x^{2}}\right|_{i, j} \approx \sum_{l=1}^{N} A_{i, l}^{(2)} f_{l, j}^{*},\left.\frac{\partial^{2} f^{*}}{\partial y^{2}}\right|_{i, j} \approx \sum_{m=1}^{M} B_{j, m}^{(2)} f_{i, m}^{*}$,

$\left.\frac{\partial^{2} f^{*}}{\partial x \partial y}\right|_{i, j} \approx \sum_{l=1}^{N} A_{i, l}^{(1)} \sum_{m=1}^{M} B_{j, m}^{(1)} f_{l, m}^{*}$

where $A_{i, j}^{(m)}$ and $B_{i, j}^{(m)}$ denote the weighting coefficients for the $m^{\text {th }}$-order derivative of the function $f^{*}(x, y)$ with respect to the $x$ and $y$ directions.

\subsection{Thermoelastic Stress-Strain Relations with Magne- tostrictive Effect}

We consider a homogeneous, orthotropic, rectangular laminated host plate that mounted with magnetostrictive layers under uniformly distributed loading and thermal effect. Typical three-layer laminate with upper surface magnetostrictive layer which is shown in Fig. (1), where a and b is the length in the $x, y$ direction of the plate, $h^{*}$ is the plate thickness, $p_{1}$ and $p_{2}$ are the in-plane distributed forces, $q$ is the applied pressure load, $\Delta T=T_{0}(x, y, t)+\frac{z}{h^{*}} T_{1}(x, y, t)$ is the temperature difference between the laminate and curing area. For simplicity, the heat conduction equation for the host laminate would be used [6].

For the plane stress in a laminated material, the in-plane stresses constitute the membrance stresses, bending stresses and thermal stresses with magnetostrictive effect for the $k^{\text {th }}$ layer are in the following equations [3]:

$$
\begin{aligned}
& \left\{\begin{array}{c}
\sigma_{x} \\
\sigma_{y} \\
\sigma_{x y}
\end{array}\right\}_{(k)}=\left[\begin{array}{lll}
\bar{Q}_{11} & \bar{Q}_{12} & \bar{Q}_{16} \\
\bar{Q}_{12} & \bar{Q}_{22} & \bar{Q}_{26} \\
\bar{Q}_{16} & \bar{Q}_{26} & \bar{Q}_{66}
\end{array}\right]_{(k)}\left\{\begin{array}{c}
\varepsilon_{x}-\alpha_{x} \Delta T \\
\varepsilon_{y}-\alpha_{y} \Delta T \\
\varepsilon_{x y}-\alpha_{x y} \Delta T
\end{array}\right\}_{(k)} \\
& -\left[\begin{array}{lll}
0 & 0 & \tilde{e}_{31} \\
0 & 0 & \tilde{e}_{32} \\
0 & 0 & \tilde{e}_{36}
\end{array}\right]_{(k)}\left\{\begin{array}{c}
0 \\
0 \\
\tilde{H}_{z}
\end{array}\right\}_{(k)}
\end{aligned}
$$

and the interlaminar shear stresses are calculated from the following equation:

$$
\begin{aligned}
& \left\{\begin{array}{c}
\sigma_{y z} \\
\sigma_{x z}
\end{array}\right\}_{(k)}=\left[\begin{array}{ll}
\bar{Q}_{44} & \bar{Q}_{45} \\
\bar{Q}_{45} & \bar{Q}_{55}
\end{array}\right]_{(k)}\left\{\begin{array}{l}
\varepsilon_{y z} \\
\varepsilon_{x z}
\end{array}\right\}_{(k)} \\
& -\left[\begin{array}{lll}
\tilde{e}_{14} & \tilde{e}_{24} & 0 \\
\tilde{e}_{15} & \tilde{e}_{25} & 0
\end{array}\right]_{(k)}\left\{\begin{array}{c}
0 \\
0 \\
\tilde{H}_{z}
\end{array}\right\}_{(k)}
\end{aligned}
$$

where $\alpha_{x}$ and $\alpha_{y}$ are the coefficients of thermal expansion, $\alpha_{x y}$ is the coefficient of thermal shear. $\bar{Q}_{i j}$ is the so called transformed reduced stiffness can be in terms of the elastic stiffness of materials and can be explained more detail by Whitney [12]. $\varepsilon_{x}, \varepsilon_{y}, \varepsilon_{x y}$ are in-plane strains and $\varepsilon_{y z}, \varepsilon_{x z}$ are shear strains in terms of displacement components and shear rotation, respectively. $\tilde{e}_{i j}$ is the transformed magnetostrictive coupling moduli. $\tilde{H}_{z}$ is the magnetic field intensity, expressed in the following equation. $\tilde{H}_{z}(x, y, t)=k_{c} \tilde{I}(x, y, t)$

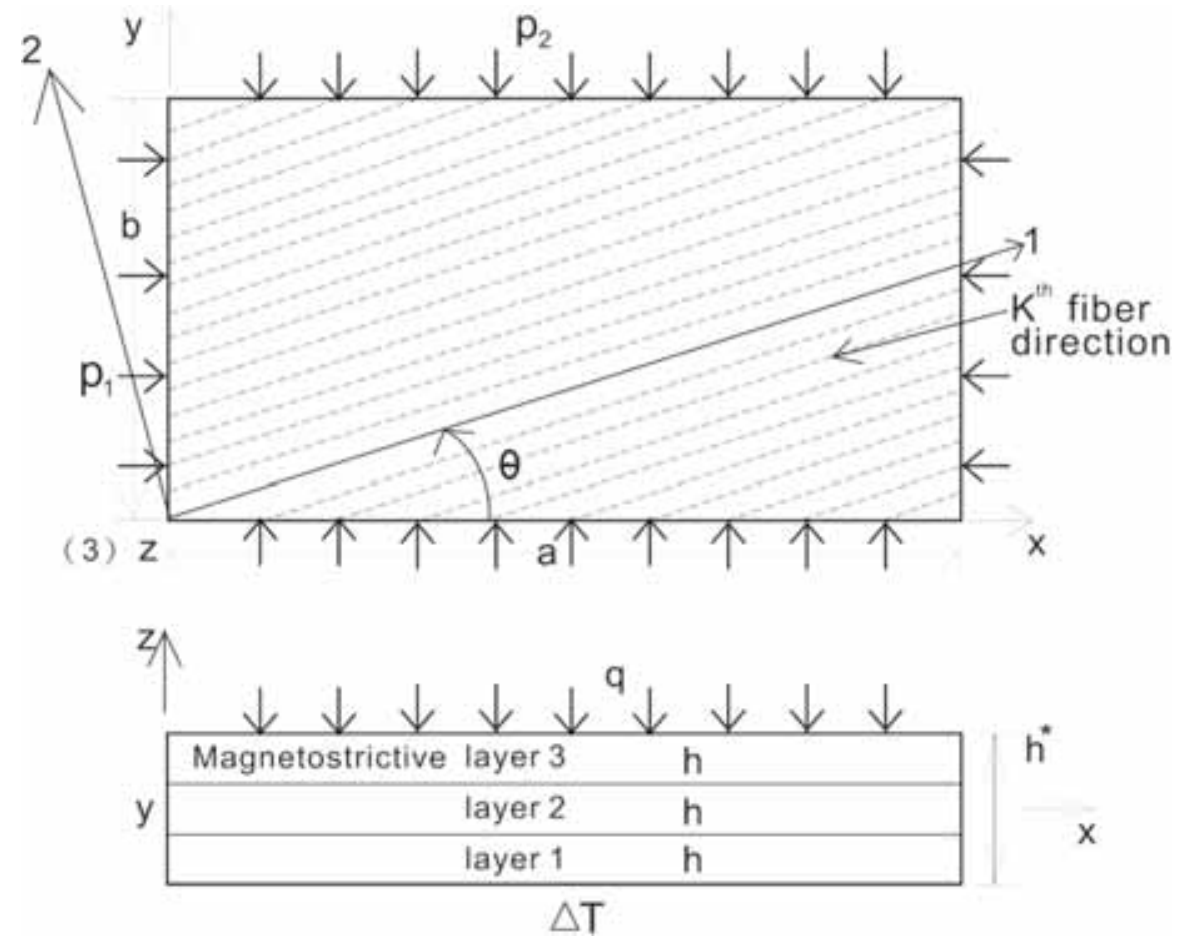

Fig. (1). Typical three-layer laminate with magnetostrictive layers. 
with velocity feedback control $\tilde{I}(x, y, t)=c(t) \frac{\partial w}{\partial t}$ where $k_{c}$ is the coil constant, $\tilde{I}(x, y, t)$ is the coil current, $c(t)$ is the control gain.

\subsection{Dynamic Equilibrium Differential Equations}

The dynamic equilibrium differential equations in terms of displacements and shear rotations included the magnetostrictive loads are expressed in the following matrix forms $[3,6,12]$ :

$$
\begin{aligned}
& {\left[\begin{array}{ccccccccc}
A_{11} & 2 A_{16} & A_{66} & A_{16} & A_{12}+A_{66} & A_{26} & 0 & 0 & 0 \\
A_{16} & A_{12}+A_{66} & A_{26} & A_{66} & 2 A_{26} & A_{22} & 0 & 0 & 0 \\
0 & 0 & 0 & 0 & 0 & 0 & -A_{55} & -2 A_{45} & -A_{44} \\
B_{11} & 2 B_{16} & B_{66} & B_{16} & B_{12}+B_{66} & B_{26} & 0 & 0 & 0 \\
B_{16} & B_{12}+B_{66} & B_{26} & B_{66} & 2 B_{26} & B_{22} & 0 & 0 & 0
\end{array}\right]}
\end{aligned}
$$

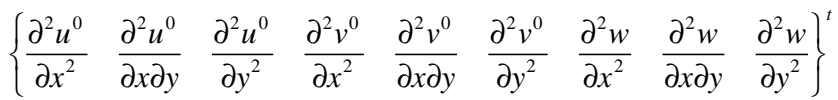

$$
\begin{aligned}
& +\left[\begin{array}{cccccc}
B_{11} & 2 B_{16} & B_{66} & B_{16} & B_{12}+B_{66} & B_{26} \\
B_{16} & B_{12}+B_{66} & B_{26} & B_{66} & 2 B_{26} & B_{22} \\
0 & 0 & 0 & 0 & 0 & 0 \\
D_{11} & 2 D_{16} & D_{66} & D_{16} & D_{12}+D_{66} & D_{26} \\
D_{16} & D_{12}+D_{66} & D_{26} & D_{66} & 2 D_{26} & D_{22}
\end{array}\right]
\end{aligned}
$$

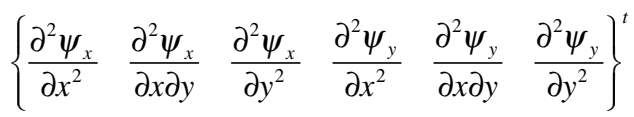

$$
\begin{aligned}
& +\left[\begin{array}{rccccc}
0 & 0 & 0 & 0 & 0 & 0 \\
0 & 0 & 0 & 0 & 0 & 0 \\
0 & 0 & -A_{55} & -A_{45} & -A_{45} & -A_{44} \\
-A_{55} & -A_{45} & 0 & 0 & 0 & 0 \\
-A_{45} & -A_{44} & 0 & 0 & 0 & 0
\end{array}\right]
\end{aligned}
$$

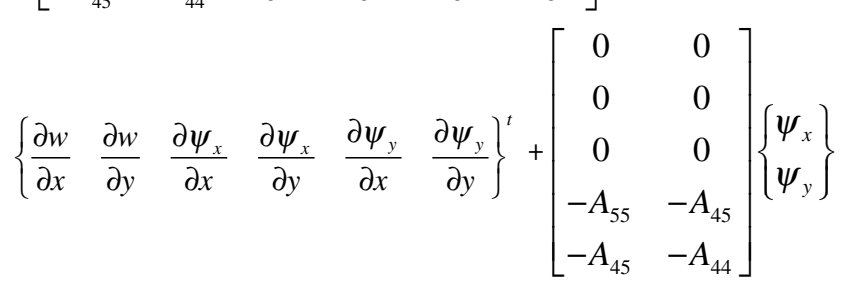

$$
\begin{aligned}
& =\left\{\begin{array}{l}
f_{1} \\
f_{2} \\
f_{3} \\
f_{4} \\
f_{5}
\end{array}\right\}+\rho\left[\begin{array}{ccc}
1 & 0 & 0 \\
0 & 1 & 0 \\
0 & 0 & -1 \\
0 & 0 & 0 \\
0 & 0 & 0
\end{array}\right]\left\{\begin{array}{l}
\frac{\partial^{2} u^{0}}{\partial t^{2}} \\
\frac{\partial^{2} v^{0}}{\partial t^{2}} \\
\frac{\partial^{2} w}{\partial t^{2}}
\end{array}\right\}+H\left[\begin{array}{llll}
0 & 0 & 1 & 0 \\
0 & 0 & 0 & 1 \\
0 & 0 & 0 & 0 \\
1 & 0 & 0 & 0 \\
0 & 1 & 0 & 0
\end{array}\right]\left\{\begin{array}{l}
\frac{\partial^{2} u^{0}}{\partial t^{2}} \\
\frac{\partial^{2} v^{0}}{\partial t^{2}} \\
\frac{\partial^{2} \psi_{x}}{\partial t^{2}} \\
\frac{\partial^{2} \psi_{y}}{\partial t^{2}}
\end{array}\right\} \\
& +I\left[\begin{array}{ll}
0 & 0 \\
0 & 0 \\
0 & 0 \\
1 & 0 \\
0 & 1
\end{array}\right]\left\{\begin{array}{c}
\frac{\partial^{2} \psi_{x}}{\partial t^{2}} \\
\frac{\partial^{2} \psi_{y}}{\partial t^{2}}
\end{array}\right\}
\end{aligned}
$$

where $f_{1}, \ldots, f_{5}$ are the expressions of thermal loads $(\bar{N}, \bar{M})$, mechanical loads $\left(p_{1}, p_{2}, q\right)$ and magnetostrictive loads $(\tilde{N}, \tilde{M})$.

$$
\begin{aligned}
& f_{1}=\frac{\partial \bar{N}_{x}}{\partial x}+\frac{\partial \bar{N}_{x y}}{\partial y}+p_{1}+\frac{\partial \tilde{N}_{x}}{\partial x}+\frac{\partial \tilde{N}_{x y}}{\partial y} \\
& f_{2}=\frac{\partial \bar{N}_{x y}}{\partial x}+\frac{\partial \bar{N}_{y}}{\partial y}+p_{2}+\frac{\partial \tilde{N}_{x y}}{\partial x}+\frac{\partial \tilde{N}_{y}}{\partial y} \\
& f_{3}=q \quad f_{4}=\frac{\partial \bar{M}_{x}}{\partial x}+\frac{\partial \bar{M}_{x y}}{\partial y}+\frac{\partial \tilde{M}_{x}}{\partial x}+\frac{\partial \tilde{M}_{x y}}{\partial y} \\
& f_{5}=\frac{\partial \bar{M}_{x y}}{\partial x}+\frac{\partial \bar{M}_{y}}{\partial y}+\frac{\partial \tilde{M}_{x y}}{\partial x}+\frac{\partial \tilde{M}_{y}}{\partial y} \\
& \left(\bar{N}_{y}, \bar{M}_{y}\right)=\int_{\frac{h^{*}}{2}}^{\frac{h^{*}}{2}}\left(\bar{Q}_{12} \alpha_{x}+\bar{Q}_{22} \alpha_{y}+\bar{Q}_{26} \alpha_{x y}\right)\left(T_{0}, z \frac{z}{h^{*}} T_{1}\right) d z \\
& \left(\bar{N}_{x y}, \bar{M}_{x y}\right)=\int_{\frac{-h^{*}}{2}}^{\frac{h^{*}}{2}}\left(\bar{Q}_{16} \alpha_{x}+\bar{Q}_{26} \alpha_{y}+\bar{Q}_{66} \alpha_{x y}\right)\left(T_{0}, z \frac{z}{h^{*}} T_{1}\right) d z \\
& \left(\tilde{N}_{x}, \tilde{M}_{x}\right)=\int_{\frac{-h}{2}}^{\frac{h^{*}}{2}} \tilde{e}_{31} \tilde{H}_{z}\left(1, z^{2}\right) d z \quad\left(\tilde{N}_{y}, \tilde{M}_{y}\right)=\int_{\frac{-h^{*}}{2}}^{\frac{h^{*}}{2}} \tilde{e}_{32} \tilde{H}_{z}\left(1, z^{2}\right) d z \\
& \left(\tilde{N}_{x y}, \tilde{M}_{x y}\right)=\int_{\frac{-h^{*}}{2}}^{\frac{h^{*}}{2}} \tilde{e}_{36} \tilde{H}_{z}\left(1, z^{2}\right) d z \\
& \left(A_{i j}, B_{i j}, D_{i j}\right)=\int_{\frac{-h}{2}}^{\frac{h^{*}}{2}} \bar{Q}_{i j}\left(1, z, z^{2}\right) d z,(i, j=1,2,6) \\
& A_{i * j}^{* *}=\int_{\frac{-h}{2}}^{\frac{h^{*}}{2}} k_{\alpha} k_{\beta} \bar{Q}_{i, j}{ }_{i j} d z,\left(i^{*}, j^{*}=4,5 ; \alpha=6-i^{*}, \beta=6-j^{*}\right) \\
& (\rho, H, I)=\int_{\frac{-h}{2}}^{\frac{h^{*}}{2}} \rho_{0}\left(1, z, z^{2}\right) d z
\end{aligned}
$$

in which $k_{\alpha}, k_{\beta}$ are the shear correction coefficients, $\rho_{0}$ is the density of ply.

\subsection{Dynamic Discretized Equations}

We apply the weighting coefficients of discretized equations (2) in the two-dimensional generalized differential qradrature (GDQ) method to discrete the differential equations (5) under the vibration of time sinusoidal displacement and temperature:

$$
\begin{aligned}
& u=\left[u^{0}(x, y)+z \psi_{x}(x, y)\right] \sin \left(\omega_{m n} t\right), \\
& v=\left[v^{0}(x, y)+z \psi_{y}(x, y)\right] \sin \left(\omega_{m n} t\right) \\
& w=w(x, y) \sin \left(\omega_{m n} t\right), \Delta T=\left[T_{0}(x, y)+\frac{z}{h^{*}} T_{1}(x, y)\right] \sin (\gamma t)
\end{aligned}
$$


where $\omega_{m n}$ is natural frequency of the plate, $\gamma$ is frequency of applied heat flux.

And the following non-dimensional parameters are introduced:

$X=x / a, Y=y / b, U=u^{0} / a, V=v^{0} / b$,

$W=10 h^{*} w /\left(\alpha_{x} \bar{T}_{1} a^{2}\right)$

under the vibra-

tion of time sinusoidal displacement and temperature.

We obtain the following dynamic discretized equations in matrix notation:

$[A M]\{S U V W\}+[B M]\{S S I X Y\}+[K E]\{S W S I\}$

$+[F Q]\{U V W S I\}=\{F\}$

where

$\{S U V W\}=\left\{\sum_{l=1}^{N} A_{i, l}^{(2)} U_{l, j} \quad \sum_{l=1}^{N} A_{i, l}^{(1)} \sum_{m=1}^{M} B_{j, m}^{(1)} U_{l, m} \sum_{m=1}^{M} B_{j, m}^{(2)} U_{i, m} \sum_{l=1}^{N} A_{i, l}^{(2)} V_{l, j}\right.$

$\left.\sum_{l=1}^{N} A_{i, l}^{(1)} \sum_{m=1}^{M} B_{j, m}^{(1)} V_{l, m} \quad \sum_{m=1}^{M} B_{j, m}^{(2)} V_{i, m} \quad \sum_{l=1}^{N} A_{i, l}^{(2)} W_{l, j} \quad \sum_{l=1}^{N} A_{i, l}^{(1)} \sum_{m=1}^{M} B_{j, m}^{(1)} W_{l, m} \quad \sum_{m=1}^{M} B_{j, m}^{(2)} W_{i, m}\right\}^{t}$

$\{S \operatorname{SIXY}\}=\left\{\sum_{l=1}^{N} A_{i, l}^{(2)} \psi_{x l, j} \sum_{l=1}^{N} A_{i, l}^{(1)} \sum_{m=1}^{M} B_{j, m}^{(1)} \psi_{x l, m} \sum_{m=1}^{M} B_{j, m}^{(2)} \psi_{x i, m}\right.$

$\left.\sum_{l=1}^{N} A_{i, l}^{(2)} \psi_{y \quad l, j} \quad \sum_{l=1}^{N} A_{i, l}^{(1)} \sum_{m=1}^{M} B_{j, m}^{(1)} \psi_{y \quad l, m} \sum_{m=1}^{M} B_{j, m}^{(2)} \psi_{y} \quad{ }_{i, m}\right\}^{t}$

$\{S W S I\}=\left\{\sum_{l=1}^{N} A_{i, l}^{(1)} W_{l, j} \quad \sum_{m=1}^{M} B_{j, m}^{(1)} W_{i, m} \quad \sum_{l=1}^{N} A_{i, l}^{(1)} \psi_{x l, j}\right.$

$\left.\sum_{m=1}^{M} B_{j, m}^{(1)} \psi_{x i, m} \quad \sum_{l=1}^{N} A_{i, l}^{(1)} \psi_{y \quad l, j} \quad \sum_{m=1}^{M} B_{j, m}^{(1)} \psi_{y \quad i, m}\right\}^{t}$

$\{U V W S I\}=\left\{U_{i, j} \quad V_{i, j} \quad W_{i, j} \quad \psi_{x i, j} \quad \psi_{y i, j}\right\}^{t}$

$\{F\}=\left\{\begin{array}{lllll}F_{1} & F_{2} & F_{3} & F_{4} & F_{5}\end{array}\right\}^{t}$

The elements of $5 \times 9$ matrix $[A M], 5 \times 6$ matrix $[B M], 5 \times 6$ matrix $[K E]$ and $5 \times 5$ matrix $[F Q]$ are as follows:

$$
\begin{aligned}
& A M_{11}=\left(A_{11} / a\right) \sin \left(\omega_{m n} t\right) \quad A M_{12}=\left(2 A_{16} / b\right) \sin \left(\omega_{m n} t\right) \\
& A M_{13}=\left(A_{66} a / b^{2}\right) \sin \left(\omega_{m n} t\right) \\
& A M_{14}=\left(A_{16} b / a^{2}\right) \sin \left(\omega_{m n} t\right) \\
& A M_{15}=\left[\left(A_{12}+A_{66}\right) / a\right] \sin \left(\omega_{m n} t\right) \\
& A M_{16}=\left(A_{26} / b\right) \sin \left(\omega_{m n} t\right) \\
& A M_{17}=A M_{18}=A M_{19}=0 A M_{21}=\left(A_{16} / a\right) \sin \left(\omega_{m n} t\right) \\
& A M_{22}=\left[\left(A_{12}+A_{66}\right) / b\right] \sin \left(\omega_{m n} t\right) \\
& A M_{23}=\left(A_{26} a / b^{2}\right) \sin \left(\omega_{m n} t\right) \\
& A M_{24}=\left(A_{66} b / a^{2}\right) \sin \left(\omega_{m n} t\right) \quad A M_{25}=\left(2 A_{26} / a\right) \sin \left(\omega_{m n} t\right) \\
& A M_{26}=\left(A_{22} / b\right) \sin \left(\omega_{m n} t\right) \quad A M_{27}=A M_{28}=A M_{29}=0 \\
& A M_{31}=A M_{32}=A M_{33}=A M_{34}=A M_{35}=A M_{36}=0
\end{aligned}
$$

$$
\begin{aligned}
& A M_{37}=\frac{-\alpha_{x} \bar{T}_{1}}{10 h^{*}} A_{55} \sin \left(\omega_{m n} t\right) \\
& A M_{38}=\frac{-\alpha_{x} \bar{T}_{1}}{10 h^{*}}\left(2 A_{45} a / b\right) \sin \left(\omega_{m n} t\right) \\
& A M_{39}=\frac{-\alpha_{x} \bar{T}_{1}}{10 h^{*}}\left(A_{44} a^{2} / b^{2}\right) \sin \left(\omega_{m n} t\right) \\
& A M_{41}=\left(B_{11} / a\right) \sin \left(\omega_{m n} t\right) \quad A M_{42}=\left(2 B_{16} / b\right) \sin \left(\omega_{m n} t\right) \\
& A M_{43}=\left(B_{66} a / b^{2}\right) \sin \left(\omega_{m n} t\right) \\
& A M_{44}=\left(B_{16} b / a^{2}\right) \sin \left(\omega_{m n} t\right) \\
& A M_{45}=\left(B_{12}+B_{66}\right)(1 / a) \sin \left(\omega_{m n} t\right) \\
& A M_{46}=\left(B_{26} / b\right) \sin \left(\omega_{m n} t\right) \quad A M_{47}=A M_{48}=A M_{49}=0 \\
& A M_{51}=\left(B_{16} / a\right) \sin \left(\omega_{m n} t\right) \\
& A M_{52}=\left(B_{12}+B_{66}\right)(1 / b) \sin \left(\omega_{m n} t\right) \\
& A M_{53}=\left(B_{26} a / b^{2}\right) \sin \left(\omega_{m n} t\right) \\
& A M_{54}=\left(B_{66} b / a^{2}\right) \sin \left(\omega_{m n} t\right) A M_{55}=\left(2 B_{26} / a\right) \sin \left(\omega_{m n} t\right) \\
& A M_{56}=\left(B_{22} / b\right) \sin \left(\omega_{m n} t\right) \quad A M_{57}=A M_{58}=A M_{59}=0 \\
& B M_{11}=\left(B_{11} / a^{2}\right) \sin \left(\omega_{m n} t\right) \quad B M_{12}=\left[2 B_{16} /(a b)\right] \sin \left(\omega_{m n} t\right) \\
& B M_{13}=\left(B_{66} / b^{2}\right) \sin \left(\omega_{m n} t\right) \quad B M_{14}=\left(B_{16} / a^{2}\right) \sin \left(\omega_{m n} t\right) \\
& B M_{15}=\left[\left(B_{12}+B_{66}\right) /(a b)\right] \sin \left(\omega_{m n} t\right) \\
& B M_{16}=\left(B_{26} / b^{2}\right) \sin \left(\omega_{m n} t\right) \\
& B M_{21}=\left(B_{16} / a^{2}\right) \sin \left(\omega_{m n} t\right) \\
& B M_{22}=\left[\left(B_{12}+B_{66}\right) /(a b)\right] \sin \left(\omega_{m n} t\right) \\
& B M_{23}=\left(B_{26} / b^{2}\right) \sin \left(\omega_{m n} t\right) \quad B M_{24}=\left(B_{66} / a^{2}\right) \sin \left(\omega_{m n} t\right) \\
& B M_{25}=\left[2 B_{26} /(a b)\right] \sin \left(\omega_{m n} t\right) \quad B M_{26}=\left(B_{22} / b^{2}\right) \sin \left(\omega_{m n} t\right) \\
& B M_{31}=B M_{32}=B M_{33}=B M_{34}=B M_{35}=B M_{36}=0 \\
& B M_{41}=\left(D_{11} / a^{2}\right) \sin \left(\omega_{m n} t\right) \quad B M_{42}=\left[2 D_{16} /(a b)\right] \sin \left(\omega_{m n} t\right) \\
& B M_{43}=\left(D_{66} / b^{2}\right) \sin \left(\omega_{m n} t\right) \quad B M_{44}=\left(D_{16} / a^{2}\right) \sin \left(\omega_{m n} t\right) \\
& B M_{45}=\left[\left(D_{12}+D_{66}\right) /(a b)\right] \sin \left(\omega_{m n} t\right) \\
& B M_{46}=\left(D_{26} / b^{2}\right) \sin \left(\omega_{m n} t\right) \\
& B M_{51}=\left(D_{16} / a^{2}\right) \sin \left(\omega_{m n} t\right) \\
& B M_{52}=\left[\left(D_{12}+D_{66}\right) /(a b)\right] \sin \left(\omega_{m n} t\right) \\
& B M_{53}=\left(D_{26} / b^{2}\right) \sin \left(\omega_{m n} t\right) \quad B M_{54}=\left(D_{66} / a^{2}\right) \sin \left(\omega_{m n} t\right) \\
& B M_{55}=\left[2 D_{26} /(a b)\right] \sin \left(\omega_{m n} t\right) \quad B M_{56}=\left(D_{22} / b^{2}\right) \sin \left(\omega_{m n} t\right) \\
& K E_{11}=-\frac{\alpha_{x} \bar{T}_{1} a^{2}}{10 h^{*}}\left[\frac{1}{a} k_{c} c(t) \sum_{k=1}^{N k} \tilde{e}_{31}\left(z_{k}-z_{k-1}\right)\right] \omega_{m n} \cos \left(\omega_{m n} t\right) \\
& K E_{12}=-\frac{\alpha_{x} \bar{T}_{1} a^{2}}{10 h^{*}}\left[\frac{1}{b} k_{c} c(t) \sum_{k=1}^{N k} \tilde{e}_{36}\left(z_{k}-z_{k-1}\right)\right] \omega_{m n} \cos \left(\omega_{m n} t\right) \\
& K E_{13}=K E_{14}=K E_{15}=K E_{16}=0
\end{aligned}
$$




$$
\begin{aligned}
& K E_{21}=-\frac{\alpha_{x} \bar{T}_{1} a^{2}}{10 h^{*}}\left[\frac{1}{a} k_{c} c(t) \sum_{k=1}^{N_{k}} \tilde{e}_{36}\left(z_{k}-z_{k-1}\right)\right] \omega_{m n} \cos \left(\omega_{m n} t\right) \\
& K E_{22}=-\frac{\alpha_{x} \bar{T}_{1} a^{2}}{10 h^{*}}\left[\frac{1}{b} k_{c} c(t) \sum_{k=1}^{N k} \tilde{e}_{32}\left(z_{k}-z_{k-1}\right)\right] \omega_{m n} \cos \left(\omega_{m n} t\right) \\
& K E_{23}=K E_{24}=K E_{25}=K E_{26}=0 \\
& K E_{31}=K E_{32}=0 \quad K E_{33}=-\left(A_{55} / a\right) \sin \left(\omega_{m n} t\right) \\
& K E_{34}=-\left(A_{45} / b\right) \sin \left(\omega_{m n} t\right) \quad K E_{35}=-\left(A_{45} / a\right) \sin \left(\omega_{m n} t\right) \\
& K E_{36}=-\left(A_{44} / b\right) \sin \left(\omega_{m n} t\right) \\
& K E_{41}=\frac{-\alpha_{x} \bar{T}_{1}}{10 h^{*}} A_{55} a \sin \left(\omega_{m n} t\right) \\
& -\frac{\alpha_{x} \bar{T}_{1} a^{2}}{10 h^{*}}\left[\frac{1}{a} k_{c} c(t) \frac{1}{3} \sum_{k=1}^{N_{k}} \tilde{e}_{31} \frac{z_{k}^{3}-z_{k-1}^{3}}{h^{*}}\right] \omega_{m n} \cos \left(\omega_{m n} t\right) \\
& K E_{42}=\frac{-\alpha_{x} \bar{T}_{1}}{10 h^{*}}\left(A_{45} a^{2} / b\right) \sin \left(\omega_{m n} t\right) \\
& -\frac{\alpha_{x} \bar{T}_{1} a^{2}}{10 h^{*}}\left[\frac{1}{b} k_{c} c(t) \frac{1}{3} \sum_{k=1}^{N k} \tilde{e}_{36} \frac{z_{k}^{3}-z_{k-1}^{3}}{h^{*}}\right] \omega_{m n} \cos \left(\omega_{m n} t\right) \\
& K E_{43}=K E_{44}=K E_{45}=K E_{46}=0 \\
& K E_{51}=\frac{-\alpha_{x} \bar{T}_{1}}{10 h^{*}} A_{45} a \sin \left(\omega_{m n} t\right) \\
& -\frac{\alpha_{x} \bar{T}_{1} a^{2}}{10 h^{*}}\left[\frac{1}{a} k_{c} c(t) \frac{1}{3} \sum_{k=1}^{N k} \tilde{e}_{36} \frac{z_{k}^{3}-z_{k-1}^{3}}{h^{*}}\right] \omega_{m n} \cos \left(\omega_{m n} t\right) \\
& K E_{52}=\frac{-\alpha_{x} \bar{T}_{1}}{10 h^{*}}\left(A_{44} a^{2} / b\right) \sin \left(\omega_{m n} t\right) \\
& -\frac{\alpha_{x} \bar{T}_{1} a^{2}}{10 h^{*}}\left[\frac{1}{b} k_{c} c(t) \frac{1}{3} \sum_{k=1}^{N k} \tilde{e}_{32} \frac{z_{k}^{3}-z_{k-1}^{3}}{h^{*}}\right] \omega_{m n} \cos \left(\omega_{m n} t\right) \\
& K E_{53}=K E_{54}=K E_{55}=K E_{56}=0 \\
& F Q_{11}=\rho \omega_{m n}^{2} a \sin \left(\omega_{m n} t\right) \quad F Q_{12}=F Q_{13}=F Q_{15}=0 \\
& F Q_{14}=H \omega_{m n}^{2} \sin \left(\omega_{m n} t\right) \\
& F Q_{22}=\rho \omega_{m n}^{2} b \sin \left(\omega_{m n} t\right) \quad F Q_{25}=H \omega_{m n}^{2} \sin \left(\omega_{m n} t\right) \\
& F Q_{21}=F Q_{23}=F Q_{24}=0 \\
& F Q_{31}=F Q_{32}=F Q_{34}=F Q_{35}=0 \\
& F Q_{33}=-\rho \omega_{m n}^{2}\left[\alpha_{x} \bar{T}_{1} a^{2} /\left(10 h^{*}\right)\right] \sin \left(\omega_{m n} t\right) \\
& F Q_{41}=H \omega_{m n}^{2} a \sin \left(\omega_{m n} t\right) \quad F Q_{42}=F Q_{43}=0 \\
& F Q_{44}=\left(-A_{55}+I \omega_{m n}^{2}\right) \sin \left(\omega_{m n} t\right) \quad F Q_{45}=-A_{45} \sin \left(\omega_{m n} t\right)
\end{aligned}
$$

$F Q_{51}=F Q_{53}=0 \quad F Q_{52}=H \omega_{m n}^{2} b \sin \left(\omega_{m n} t\right)$

$F Q_{54}=-A_{45} \sin \left(\omega_{m n} t\right)$

$F Q_{55}=\left(-A_{44}+I \omega_{m n}^{2}\right) \sin \left(\omega_{m n} t\right)$

in which $F_{1}, \ldots, F_{5}$ are represented in the following discretized equation:

$$
\begin{aligned}
& F_{1}=\left(\frac{1}{a} \sum_{l=1}^{N} A_{i, l}^{(1)} \bar{N}_{x l, j}+\frac{1}{b} \sum_{m=1}^{M} B_{j, m}^{(1)} \bar{N}_{x y i, m}\right) \sin (\gamma t)+p_{1 i, j} \\
& F_{2}=\left(\frac{1}{a} \sum_{l=1}^{N} A_{i, l}^{(1)} \bar{N}_{x y l, j}+\frac{1}{b} \sum_{m=1}^{M} B_{j, m}^{(1)} \bar{N}_{y i, m}+p_{2 i, j}\right) \sin (\gamma t) \\
& F_{3}=q_{i, j} \\
& F_{4}=\left(\frac{1}{a} \sum_{l=1}^{N} A_{i, l}^{(1)} \bar{M}_{x l, j}+\frac{1}{b} \sum_{m=1}^{M} B_{j, m}^{(1)} \bar{M}_{x y i, m}\right) \sin (\gamma t) \\
& F_{5}=\left(\frac{1}{a} \sum_{l=1}^{N} A_{i, l}^{(1)} \bar{M}_{x y l, j}+\frac{1}{b} \sum_{m=1}^{M} B_{j, m}^{(1)} \bar{M}_{y i, m}\right) \sin (\gamma t)
\end{aligned}
$$

in which $p_{1}$ and $p_{2}$ are the in-plane distributed forces, $q$ is the applied pressure load. The force resultants $\bar{N}_{x}, \bar{N}_{x y}, \bar{N}_{y}$ and moment resultants $\bar{M}_{x}, \bar{M}_{x y}, \bar{M}_{y}$ are expressed as follows:

$$
\begin{aligned}
& \bar{N}_{x}=\bar{N}_{x}^{T}, \bar{N}_{x y}=\bar{N}_{x y}^{T}, \bar{N}_{y}=\bar{N}_{y}^{T}, \bar{M}_{x}=\bar{M}_{x}^{T}, \bar{M}_{x y}=\bar{M}_{x y}^{T}, \\
& \bar{M}_{y}=\bar{M}_{y}^{T}
\end{aligned}
$$

in which $\left\{\bar{N}^{T}\right\}$ is the thermal force resultant, $\left\{\bar{M}^{T}\right\}$ is the thermal moment resultant.

\section{SOME NUMERICAL RESULTS AND DISCUS- SIONS}

We consider the cross-ply laminates including shear deformation under four sides simply supported. The elastic modules of the typical host material and Terfenol-D magnetostrictive material are listed in the following Table $1[3,6]$.

The value of shear correction coefficient is $K_{\alpha} K_{\beta}=5 / 6$. For the simplification the typical host material conductivity $\kappa=0.002963 B t u /\left(\sin { }^{\circ} F\right)$, specific heat $C_{v}=0.216 \mathrm{Btu} /\left(\mathrm{lbm}^{\circ} \mathrm{F}\right) \quad$ are used to calculate $K=\kappa /\left(\rho C_{v}\right)$ value, and find some of the lowest frequency $\gamma$ of applied heat flux and vibration frequency $\omega_{11}$ of plate,

Table 1. Material Properties of Typical Host and Terfenol-D

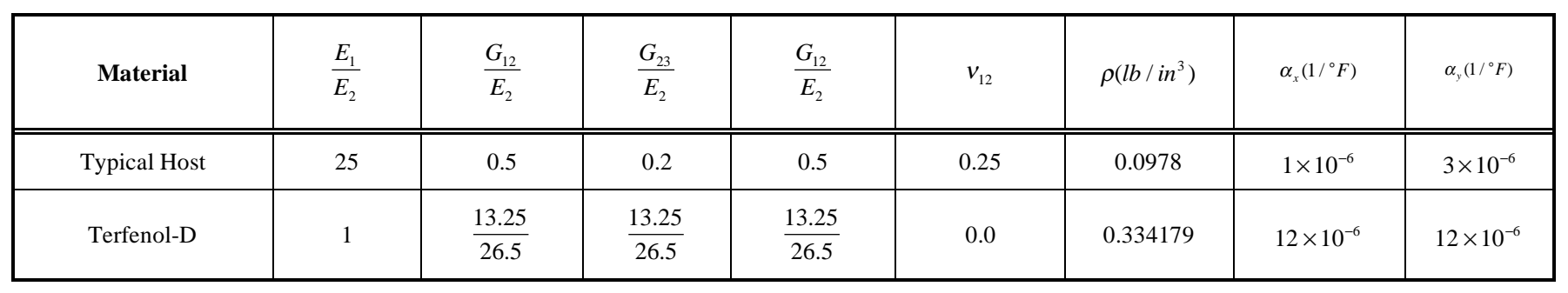


at time $t=0.003 \mathrm{~s}, 1 \mathrm{~s}, 2 \mathrm{~s}, \ldots, 9 \mathrm{~s}$, for $a / b=1$, three-layer $\left(0^{\circ} \mathrm{m} / 90^{\circ} / 0^{\circ}\right)$ laminate as shown in Fig. (2).

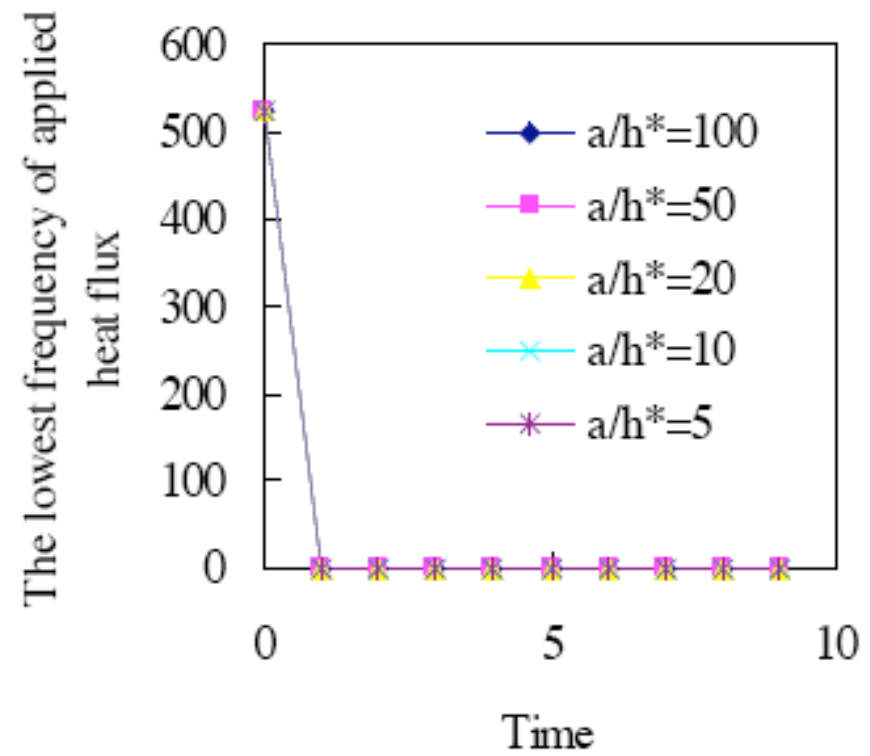

Fig. (2a). The lowest frequency $\gamma$ of three-layer $\left(0^{\circ} \mathrm{m} / 90^{\circ} / 0^{\circ}\right)$ laminate.

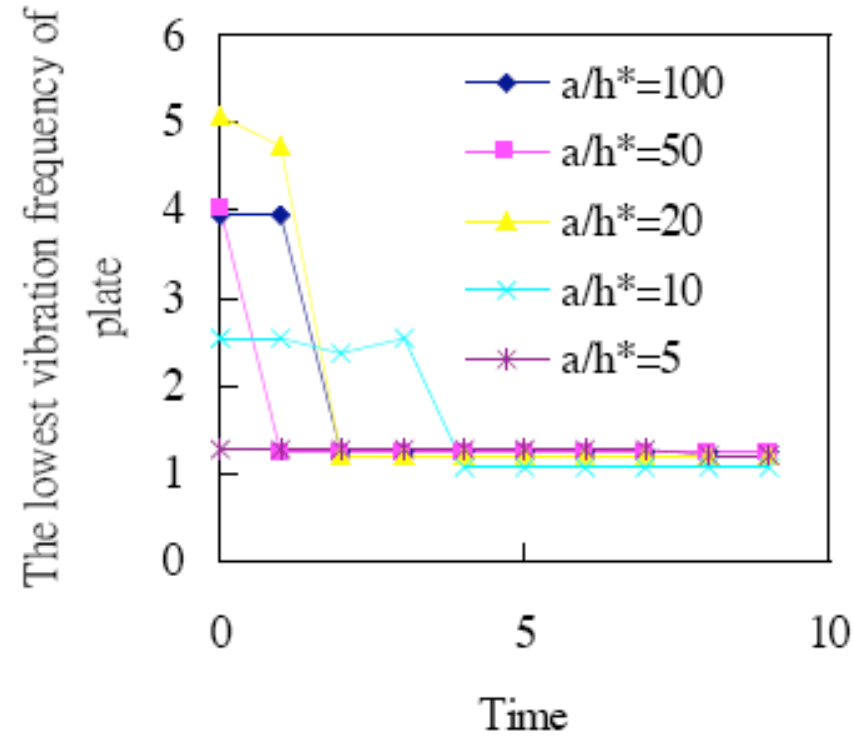

Fig. (2b). The lowest vibration frequency $\omega_{11}$ of three-layer $\left(0^{\circ} \mathrm{m} / 90^{\circ} / 0^{\circ}\right)$ laminate.

The magnetostrictive coupling moduli is $e_{31}=e_{32}=E^{m} d^{m}$ and $E^{m}=26.5 \mathrm{GPa}, d^{m}=1.67 \times 10^{-8} m A^{-1}$ for Terfenol-D. We use the following coordinates for the grid points:

$x_{i}=0.5\left[1-\cos \left(\frac{i-1}{N-1} \pi\right)\right] a, i=1,2, \ldots, N$

$y_{j}=0.5\left[1-\cos \left(\frac{j-1}{M-1} \pi\right)\right] b, j=1,2, \ldots, M$

Firstly, we make the studies of dynamic convergence of center deflection amplitude $W(a / 2, b / 2)$ in the thermal vibration of sinusoidal temperature only $\left(T_{0}=0\right.$, $\left.\bar{T}_{1}=1.0^{\circ} \mathrm{F}, p_{1}=p_{2}=q=0\right)$ at time $6 \mathrm{sec}, m=n=1$ mode shape, with $k_{c} c(t)=10^{8}$, aspect ratio $a / b=0.5,1.0$, and 2.0, side-to-thickness ratio $a / h^{*}=100,50,20,10$ and 5. Fig. (3) shows that $W(a / 2, b / 2)$ in the grid point $N \times M=$ $9 \times 9,11 \times 11$ and $13 \times 13$ of GDQ method for the upper surface magnetostrictive layer of the three-layer $\left(0^{\circ} \mathrm{m} / 90^{\circ} / 0^{\circ}\right)$ laminated plate. We find that $W(a / 2, b / 2)$ is in good convergence in grid point $N \times M=13 \times 13$.

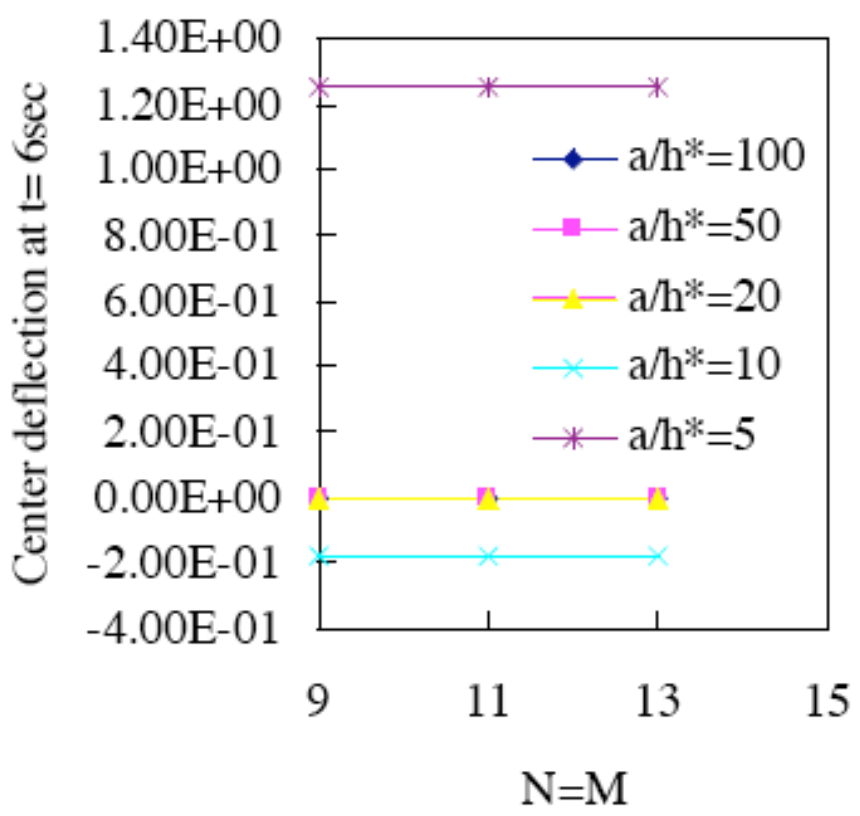

Fig. (3a). Convergence for $a / b=0.5$, three-layer $\left(0^{\circ} \mathrm{m} / 90^{\circ} / 0^{\circ}\right)$ laminated plate.

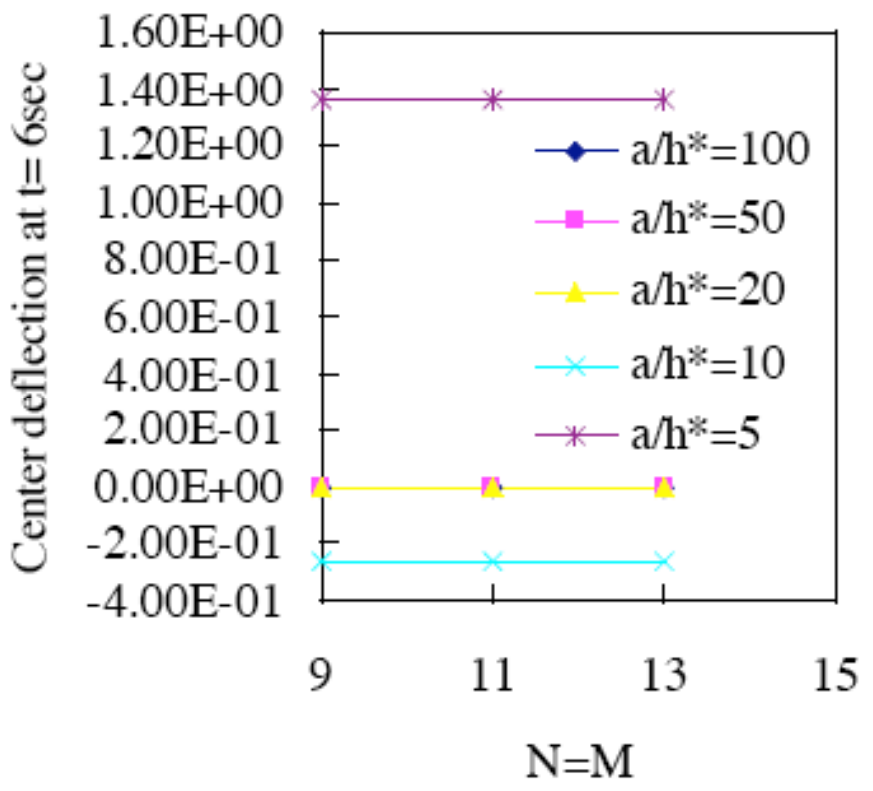

Fig. (3b). Convergence for $a / b=1.0$, three-layer $\left(0^{\circ} \mathrm{m} / 90^{\circ} / 0^{\circ}\right)$ laminated plate.

Fig. (4) shows that $W(a / 2, b / 2)$ in the grid point $N \times M=9 \times 9,11 \times 11$ and $13 \times 13$ of GDQ method for the upper and lower surface magnetostrictive layers of the ten- 
layer $\left(0^{\circ} \mathrm{m} / 90^{\circ} / 0^{\circ} / 90^{\circ} / 0^{\circ}\right)_{s}$ symmetrical laminated plate. The superscript of $\mathrm{m}$ denotes magnetostrictive layer, the subscript $\mathrm{s}$ denotes the symmetric of laminate. We find that the $13 \times 13$ grid point have the convergence result and used further in the GDQ analyses of time responses for deflection and stress. We used the $k_{c} c(t)$ values to control and reduce the amplitude of center deflection $W(a / 2, b / 2)$.

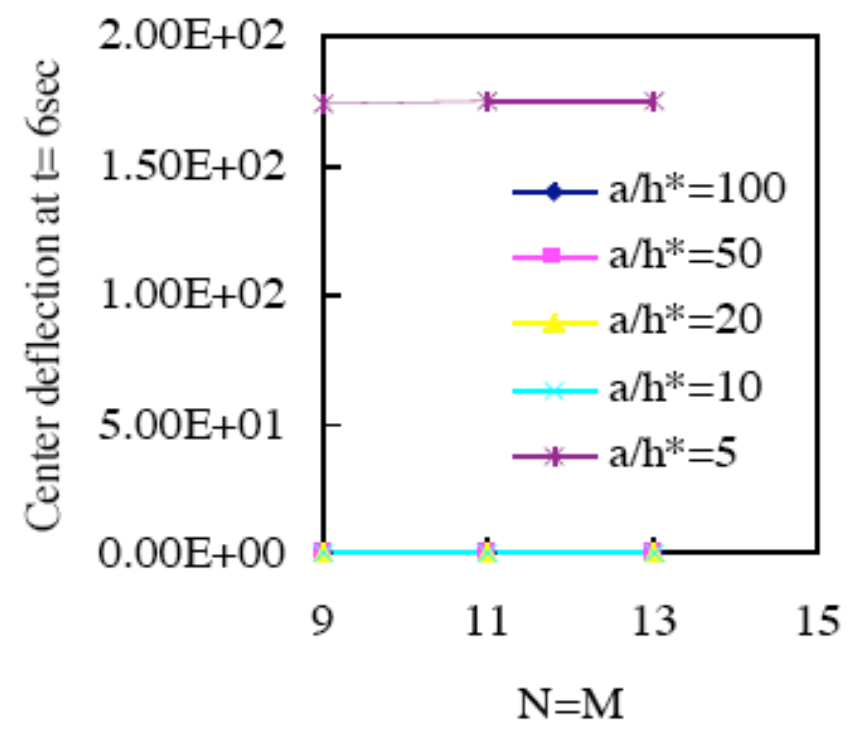

Fig. (3c). Convergence for $a / b=2.0$, three-layer $\left(0^{\circ} \mathrm{m} / 90^{\circ} / 0^{\circ}\right)$ laminated plate.

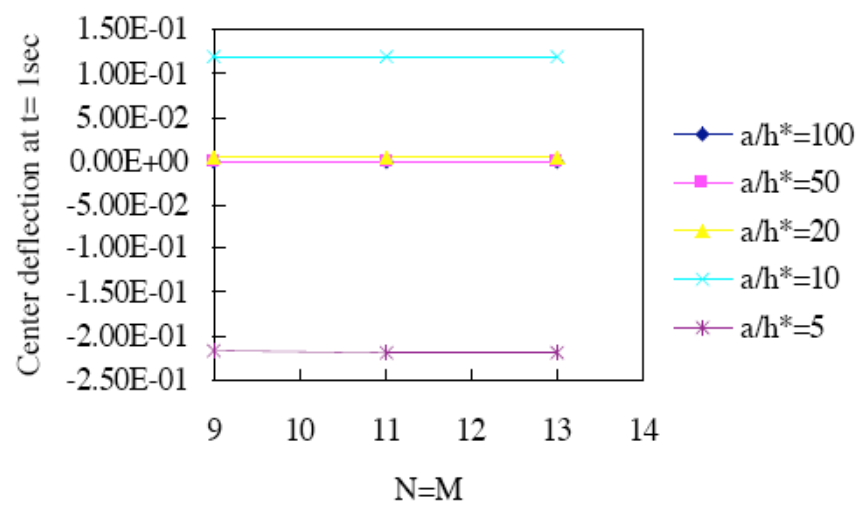

Fig. (4a). Convergence for $a / b=0.5,\left(0^{\circ} \mathrm{m} / 90^{\circ} / 0^{\circ} / 90^{\circ} / 0^{\circ}\right)_{s}$ laminated plate.

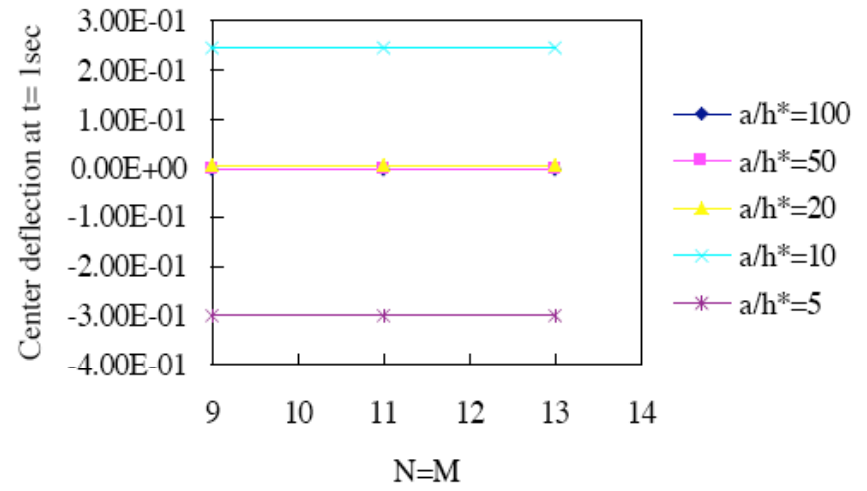

Fig. (4b). Convergence for $a / b=1.0,\left(0^{\circ} \mathrm{m} / 90^{\circ} / 0^{\circ} / 90^{\circ} / 0^{\circ}\right)_{s}$ laminated plate.

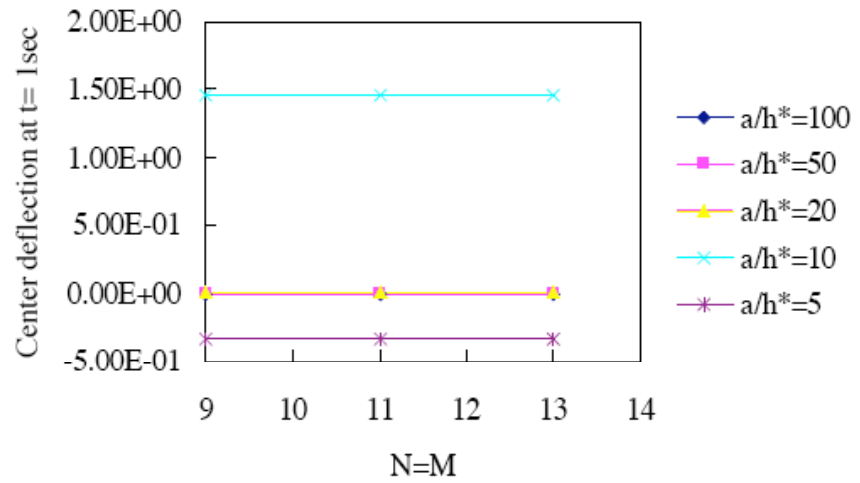

Fig. (4c). Convergence for $a / b=2.0,\left(0^{\circ} \mathrm{m} / 90^{\circ} / 0^{\circ} / 90^{\circ} / 0^{\circ}\right)_{s}$ laminated plate.

Figs. $(5,6)$ show that the $k_{c} c(t)$ values with time for thick laminated plate $a / h^{*}=10$ and thin laminated $a / h^{*}=100$, in three-layer and ten-layer magnetostrictive layer of laminate, respectively.

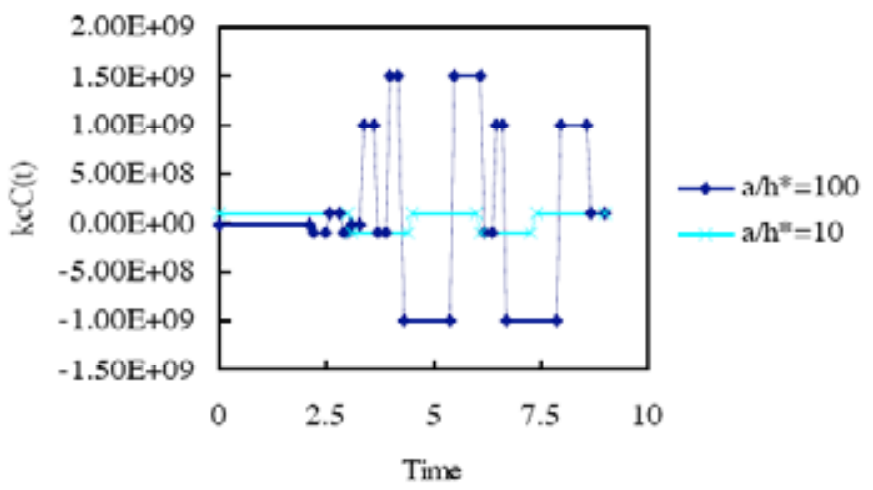

Fig. (5). $k_{c} c(t)$ values $v s$ time $t(\mathrm{sec})$ in $\left(0^{\circ} \mathrm{m} / 90^{\circ} / 0^{\circ}\right)$ laminated plate.

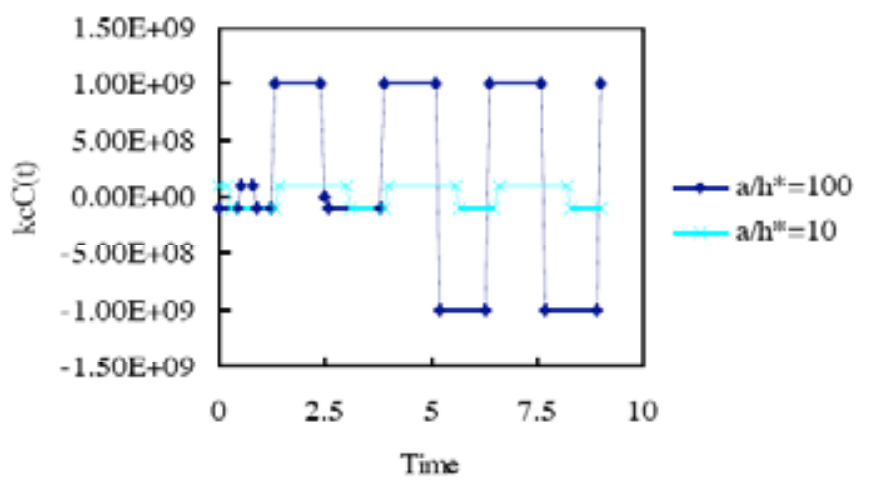

Fig. (6). $k_{c} c(t)$ values vs time $t(\mathrm{sec})$ in $\left(0^{\circ} \mathrm{m} / 90^{\circ} / 0^{\circ} / 90^{\circ} / 0^{\circ}\right) s$ laminated plate.

Fig. (7) shows that time response of the non-dimensional transverse center deflection amplitude $W(a / 2, b / 2)$ with and without $k_{c} c(t)$ values at $a / h^{*}=10$ and $a / h^{*}=100$, respectively, for three-layer $\left(0^{\circ} \mathrm{m} / 90^{\circ} / 0^{\circ}\right)$ laminated plate, $a / b=1, \quad N \times M=13 \times 13, \quad m=1, n=1, \quad q=0, \bar{T}_{1}=1$, $p_{1}=p_{2}=0$ under shear effect. We find that the values of the center deflection amplitude $W(a / 2, b / 2)$ with $k_{c} c(t)$ 
are smaller than the values of $W(a / 2, b / 2)$ without $k_{c} c(t)$. The amplitude $W(a / 2, b / 2)$ can be controlled and adjusted to a desired smaller value by using a suitable $k_{c} c(t)$ value, especially at time $0.003 \mathrm{~s}, 1.2 \mathrm{~s}, 5.9 \mathrm{~s}$ and $8.8 \mathrm{~s}$ in Fig. (7a) with thick plate $a / h^{*}=10$ of the GDQ method. But in Fig. (7b) with thin plate $a / h^{*}=100$ of the GDQ method, the variations are small, except at time $0.003 \mathrm{~s}$ and $0.8 \mathrm{~s}$.

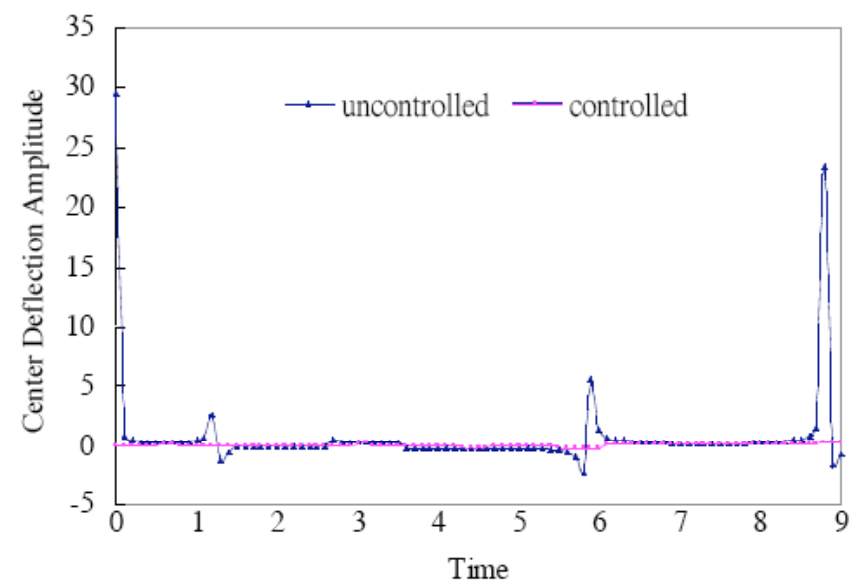

Fig. (7a). Thick, three-layer laminated magnetostrictive plate.

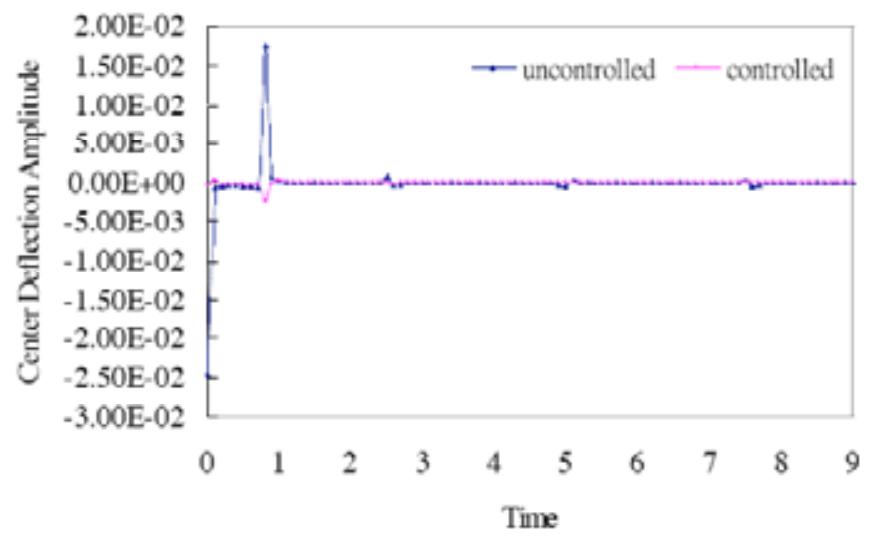

Fig. (7b). Thin, three-layer laminated magnetostrictive plate.

Fig. (8). shows that time response of the dominated nondimensional stress $\bar{\sigma}_{x}=\sigma_{x} h^{*} /\left(\alpha_{x} \overline{T_{1}} a E_{2}\right)$ at center position of lower surface $Z=-0.5 h^{*}$ with and without $k_{c} c(t)$ values as the analyses of deflection $W(a / 2, b / 2)$ at $a / h^{*}=10$ and $a / h^{*}=100$, respectively, for three-layer $\left(0^{\circ} \mathrm{m} / 90^{\circ} / 0^{\circ}\right)$ laminate, $a / b=1, N \times M=13 \times 13, m=1, n=1, q=0, \bar{T}_{1}=1$, $p_{1}=p_{2}=0$ under shear effect. We find that the amplitudes of the stress $\bar{\sigma}_{x}$ do not become the smaller values under the controlled $W(a / 2, b / 2)$ condition in Fig. (8a) with plate $a / h^{*}=10$ of GDQ method. But in Fig. (8b) with thin plate $a / h^{*}=100$ of the GDQ method, the variations are small.

Fig. (9) shows that time response of the non-dimensional transverse center deflection amplitude $W(a / 2, b / 2)$ with and without $k_{c} c(t)$ values at $a / h^{*}=10$ and $a / h^{*}=100$, respectively, for ten-layer $\left(0^{\circ} \mathrm{m} / 90^{\circ} / 0^{\circ} / 90^{\circ} / 0^{\circ}\right)_{s}$ symmet- ric laminate, $\quad a / b=1, \quad N \times M=13 \times 13, \quad m=1, n=1$, $q=0, \bar{T}_{1}=1, p_{1}=p_{2}=0$ under shear effect. We find that the values of the center deflection amplitude $W(a / 2, b / 2)$ with $k_{c} c(t)$ are smaller than the values of $W(a / 2, b / 2)$ without $k_{c} c(t)$. The amplitude $W(a / 2, b / 2)$ can be controlled and adjusted to a desired smaller value by using a suitable $k_{c} c(t)$ value, especially at time $0.003 \mathrm{~s}, 2.6 \mathrm{~s}, 5.2 \mathrm{~s}$ and $7.8 \mathrm{~s}$ in Fig. (9a) with thick plate $a / h^{*}=10$ of the GDQ method. But in Fig. (9b) with thin plate $a / h^{*}=100$ of the GDQ method, the variations are small, except at time 5.1s.

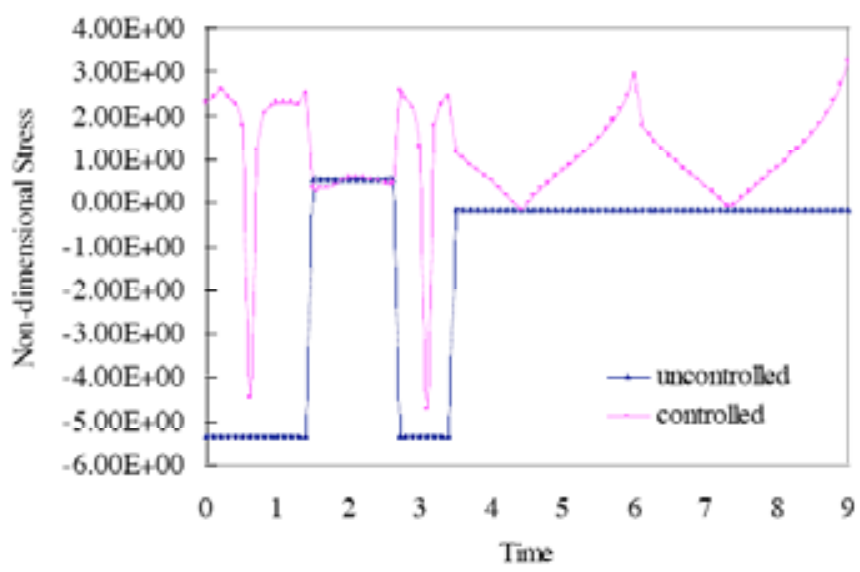

Fig. (8a). Thick, three-layer laminated magnetostrictive plate.

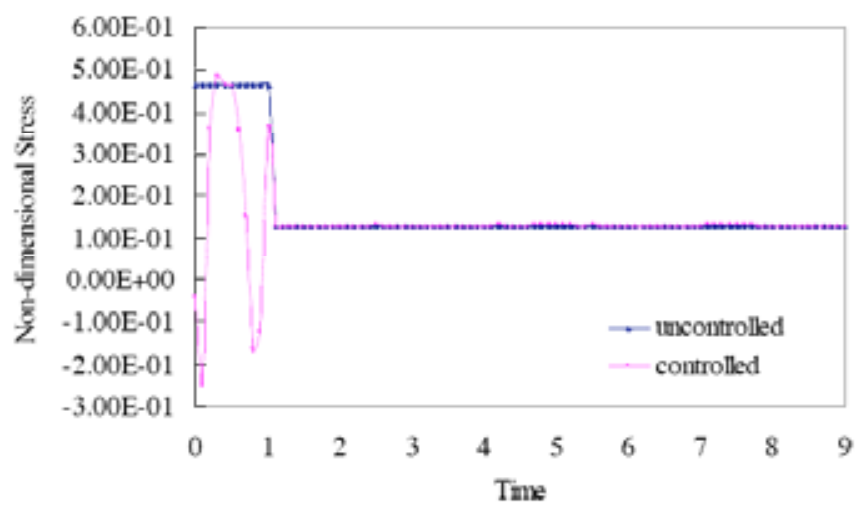

Fig. (8b). Thin, three-layer laminated magnetostrictive plate.

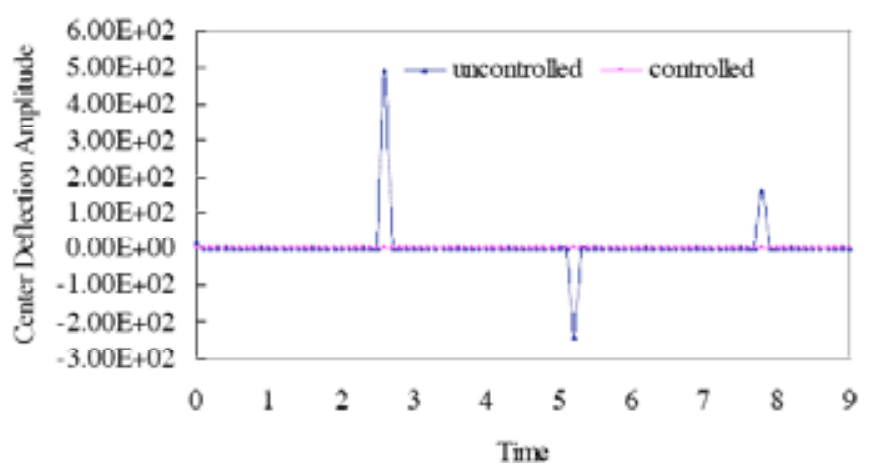

Fig. (9a). Thick, ten-layer laminated magnetostrictive plate. 


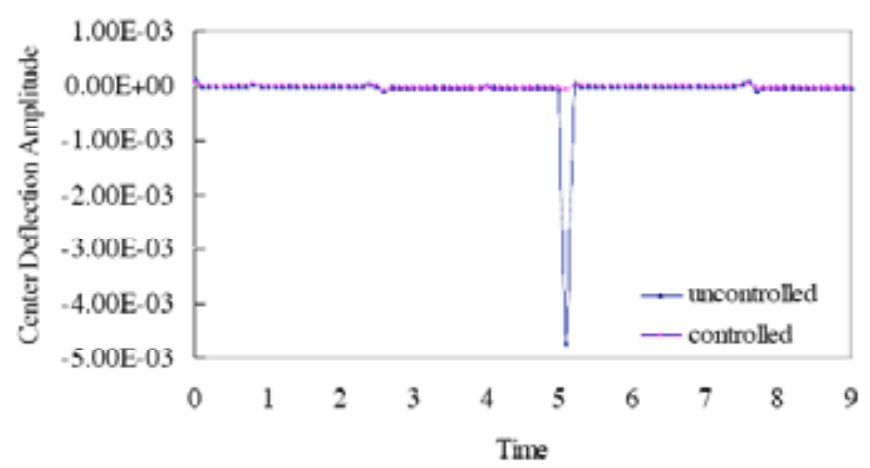

Fig. (9b). Thin, ten-layer laminated magnetostrictive plate.

Fig. (10) shows that time response of the dominated nondimensional stress $\bar{\sigma}_{y}=\sigma_{y} h^{*} /\left(\alpha_{x} \bar{T} a E_{2}\right)$ at center position of lower surface $Z=0.5 h^{*}$ with and without $k_{c} c(t)$ values as the analyses of deflection $W(a / 2, b / 2)$ at $a / h^{*}=10$ and $a / h^{*}=100$, respectively, for ten-layer $\left(0^{\circ} \mathrm{m} / 90^{\circ} / 0^{\circ} / 90^{\circ} / 0^{\circ}\right)_{s}$ symmetric laminate, $a / b=1, N \times M=13 \times 13, m=1, n=1$, $q=0, \bar{T}_{1}=1, p_{1}=p_{2}=0$ under shear effect. We find that the amplitudes of the stress $\bar{\sigma}_{y}$ are almost the same value in controlled and uncontrolled $W(a / 2, b / 2)$ condition of thick plate $a / h^{*}=10$ and thin plate $a / h^{*}=100$ by the GDQ method.

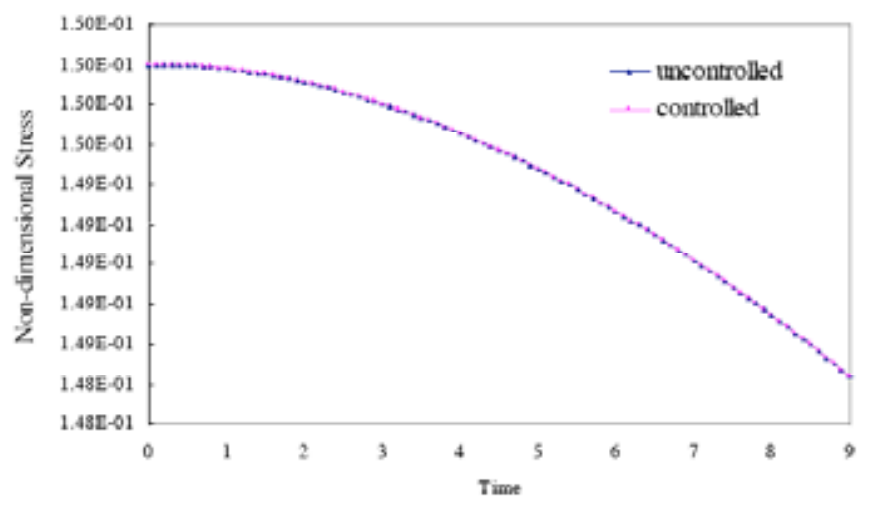

Fig. (10a). thick, ten-layer laminated magnetostrictive plate.

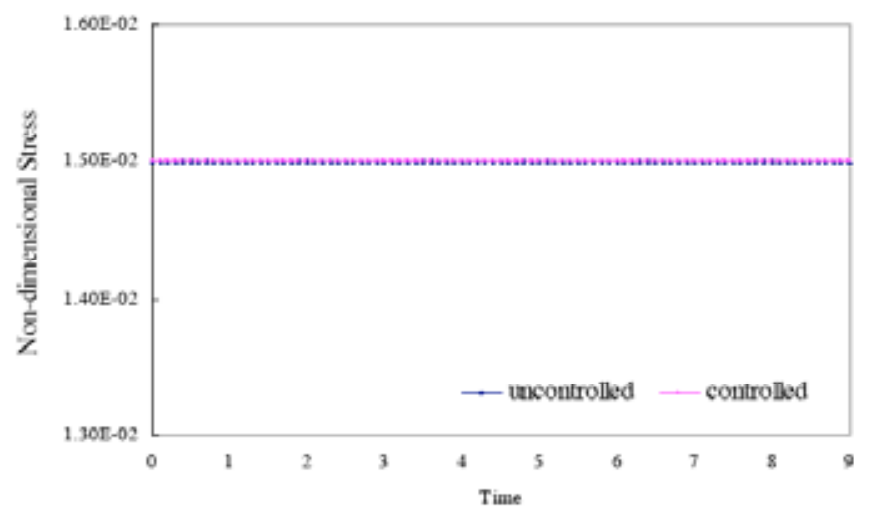

Fig. (10b). Thin, ten-layer laminated magnetostrictive plate.

\section{CONCLUSIONS}

(a) The numerical GDQ calculations provides an efficient method to compute the deflection and stress in the cross ply laminated plate with magnetostrictive layer subjected to thermal vibration of sinusoidal temperature including shear deformation. (b) The amplitude of transverse center deflection $W(a / 2, b / 2)$ can be controlled to a smaller desirable value with suitable velocity feedback gain $k_{c} c(t)$ value, especially in the thick plate $a / h^{*}=10$ by using the GDQ method. (c) The amplitude of the dominated stress $\bar{\sigma}_{x}$ is not become a smaller values under the corresponding controlled $W(a / 2, b / 2)$ condition for three-layer $\left(0^{\circ} \mathrm{m} / 90^{\circ} / 0^{\circ}\right)$ laminate in the thick plate $a / h^{*}=10$ of the GDQ method. But the amplitudes of the dominated stress $\bar{\sigma}_{y}$ are almost the same value in the corresponding controlled and uncontrolled $W(a / 2, b / 2)$ condition for ten-layer $\left(0^{\circ} \mathrm{m} / 90^{\circ} / 0^{\circ} / 90^{\circ} / 0^{\circ}\right)_{s}$ symmetric laminate of the GDQ method. (d) The GDQ method provide the less grid points $(N \times M=13 \times 13)$ and less computational time to get the numerical solutions of deflection and stress for magnetostrictive layer laminates.

\section{ACKNOWLEDGEMENTS}

The completion of this study was made possible by a grant NSC-95-2221-E-164-008 from National Science Council, Taiwan, ROC.

\section{REFERENCES}

[1] Pradhan SC. Vibration suppression of FGM shells using embedded magnetostrictive layers. Int J Solids Struct 2005; 42: 2465-2488.

[2] Wojciechowski S. New trends in the development of mechanical engineering materials. J Mater Process Tech 2000; 106: 230-235.

[3] Lee SJ, Reddy JN. Non-linear response of laminated composite plates under thermomechanical loading. Int $\mathrm{J}$ Non-Linear Mech 2005; 40: 971-985.

[4] Ramirez F, Heyliger PR, Pan E. Free vibration response of twodimensional magneto-electro-elastic laminated plates. J Sound Vibrat 2006; 292: 626-644.

[5] Buchanan GR. Layered versus multiphase magneto-electro-elastic composites. Composites Part B. Eng 2004; 35: 413-420.

[6] Hong CC, Jane $\mathrm{KC}$, Shear deformation in thermal bending analysis of laminated plates by the GDQ method. Mech Res Commun 2003; 30: $175-186$

[7] Hong CC, Liao HW, Lee LT, Ke JB, Jane KC, Thermally induced vibration of a thermal sleeve with the GDQ method. Int J Mech Sci 2005; 47: 1789-1806.

[8] Hong CC, Liao HW, Lee LT, Ke JB, Jane KC, Thermally induced vibration of a thermal sleeve with the GDQ method. Int J Mech Sci 2005; 47: 1789-1806.

[9] Hong CC, Liao HW, Jane KC. Numerical analyses of piezoelectric materials with the GDQ method. Comput Mat Sci 2006; 35: 391398.

[10] Bert CW, Jang SK, Striz AG. Nonlinear bending analysis of orthotropic rectangular plates by the method of differential quadrature. Comput Mech 1989; 5: 217-226.

[11] Shu C, Du H. Implementation of clamped and simply supported boundary conditions in the GDQ free vibration analyses of beams and plates. Int J Solds Struct 1997; 34: 819-835.

[12] Whitney JM. Structural analysis of laminated anisotropic plates. Lancaster, PA, USA: Technomic Publishing Company, Inc., 1987. 